\title{
Consensus for distributed EM-based clustering in WSNs
}

\author{
Silvana Silva Pereira*, Sergio Barbarossa ${ }^{\dagger}$, Alba Pagès-Zamora* \\ *SPCOM Group, Universitat Politècnica de Catalunya-Barcelona Tech (UPC) \\ C/Jordi Girona 1-3 08034, Barcelona, Spain. E-mail: \{silvana.silva,alba.pages\} @upc.edu. \\ $\dagger$ INFOCOM Dpt., Sapienza University of Rome \\ Via Eudossiana 18, 00184 Rome, Italy. E-mail: sergio@infocom.uniroma1.it
}

\begin{abstract}
A distributed EM algorithm with consensus is proposed for density estimation and clustering using WSNs in the presence of mixtures of Gaussians. The EM algorithm is a general framework for maximum likelihood estimation in hidden variable models, usually implemented in a central node with global information of the network. The average consensus algorithm is a simple robust scheme for computing averages in a distributed manner. In this contribution, we run a distributed EM algorithm where the nodes obtain global knowledge of the statistics through consensus with local information exchange only in a WSN with instantaneous random links. Starting from a set of initial values, the nodes are able to compute the complete statistics of a mixture of Gaussians and classify into clusters according to the sensed density using a simple decision rule. A trade off between power consumption and final accuracy of the estimates is established through simulations.
\end{abstract}

\section{INTRODUCTION}

The term clustering refers to a classification of data into subsets or clusters, where the observations share some common characteristics. The task of finding a structure in a set of data for classification involves the process of comparing the observations according to some distance measurement (e.g. euclidean, manhattan, norm) such that members of the same group are close to each other in some sense. The advantages of organizing data in clusters are manifold. For instance, the communication protocols in a hierarchical WSN structured in clusters can be strongly simplified. Mixtures of Gaussians are linear combinations of Gaussian densities in multidimensional spaces, useful in different fields like statistical analysis, pattern recognition or machine learning, but also useful to cluster data [1], [2]. A natural choice for classification and density estimation in presence of mixtures of Gaussians with hidden variables is the expectation-maximization (EM) algorithm, an iterative method for computing maximum likelihood (ML) estimates. The EM algorithm is usually implemented in a central node having access to information of the whole network, although some contributions dealing with decentralized implementations can be found in literature. For instance, a distributed EM algorithm where a path is built through all the nodes in a sensor network to estimate the Gaussian densities

This work has been partially funded by the Spanish Ministry of Education and Science and FEDER funds (CONSOLIDER CSD2008-00010 COMONSENS and TEC2006-06481) and by the Catalan Government (2009SGR01236 AGAUR). using an incremental scheme is proposed in [3]. Moreover, the authors in [4] extend the work in [3] for differentiable and logconcave pdf's and use the method of multipliers in a network structured in clusters where so called bridge nodes (cluster heads) impose consensus in neighboring nodes to force the convergence of the whole network to the same estimates. In this contribution, we propose a different approach for density estimation and clustering in WSNs in the presence of mixtures of Gaussians, where the estimates at each iteration of the EM algorithm are obtained using the consensus algorithm. The nodes are able to reach an agreement regarding the complete statistics of the data and after convergence they are able to classify into clusters according to the sensed density. The EM algorithm may converge either to a local maximum, a global maximum or a saddle point of the log-likelihood function, whereas the consensus algorithm converges to a common value provided that the network is connected in average over time. In other words, the consensus algorithm has the advantage of being robust w.r.t. random packet loss, which affects only the convergence rate but not the convergence itself. We show that our implementation is assured to converge for properly initialized consensus variables and derive the error threshold minimizing the total number of iterations through simulations in a WSN with random symmetric links. The paper is organized as follows. In section II we present the notation and basic concepts of graph theory. In section III we introduce the conventional EM algorithm and in section IV we present the distributed EM with consensus for clustering. Simulations and conclusions are included in sections V and VI respectively.

\section{PREliminaries}

Notation: Scalar values, vectors and matrices are denoted with lower case letters, bold lower case letters and bold capital letters $x, \mathbf{x}$ and $\mathbf{X}$ respectively, whereas sets are denoted with calligraphic letters $\mathcal{X}$. The bar (.) denotes mean, $(.)^{T}$ denotes transpose and vec(.) denotes the vec operator, which stacks the column vectors of an $M \times N$ matrix into a column vector of length $M \times N$.

Graph theory concepts: The information flow among the nodes of a random network is described by a graph $\mathcal{G}(l)=$ $\{\mathcal{V}, \mathcal{E}(l)\}$, where $\mathcal{E}(l)$ is the set of edges $e_{i j}$ at time $l$, for all $i, j \in\{1, \cdots, N\}$ and $\mathcal{V}$ is the constant set of nodes [5]. 
$e_{i j} \in \mathcal{E}(l)$ with probability $0 \leqslant p_{i j} \leqslant 1$, and each link has a weight equal to 1 if $e_{i j} \in \mathcal{E}(l)$ and equal to 0 otherwise. We assume $e_{i j}=e_{j i}$ and $e_{i i}=0$ for all $i$. The set of nodes sending information to node $i$ at time $l$ is denoted $\mathcal{N}_{i}(l)=\left\{j \in \mathcal{V}: e_{i j} \in \mathcal{E}(l)\right\}$. The instantaneous adjacency matrix $\mathbf{A}(l) \in \mathbb{R}^{N \times N}$ of $\mathcal{G}(l)$ is random with statistically independent entries $[\mathbf{A}(l)]_{i j}=1$ with probability $p_{i j}$ and $[\mathbf{A}(l)]_{i j}=0$ with probability $1-p_{i j}$ and symmetric mean $\overline{\mathbf{A}}=\mathbf{P}$ where $\mathbf{P}$ is the probability matrix. The degree matrix $\mathbf{D}(l) \in \mathbb{R}^{N \times N}$ is a diagonal matrix with entries $[\mathbf{D}(l)]_{i i}=\sum_{j=1}^{N}[\mathbf{A}(l)]_{i j}$, and the instantaneous Laplacian is defined as $\mathbf{L}(l)=\mathbf{D}(l)-\mathbf{A}(l)$. Due to the random nature of $\mathbf{A}(l), \mathbf{L}(l)$ is random. By construction, the smallest eigenvalue of $\mathbf{L}(l)$ in magnitude is $\lambda_{1}(\mathbf{L}(l))=0$, arranged in increasing order by convention. If $\mathcal{G}(l)$ is connected, $\lambda_{1}(\mathbf{L}(l))$ has algebraic multiplicity one and $\mathbf{L}(l)$ is an irreducible matrix [6].

\section{EM ALGORITHM FOR DENSITY ESTIMATION}

Mixtures of Gaussians: Assume a set of wireless sensor nodes deployed to sense variables from a mixture of Gaussians. A D-dimensional observation vector is formed with variables drawn from a set of $K$ Gaussian densities with probability distribution $\mathcal{N}\left(\mathbf{x} \mid \boldsymbol{\mu}_{k}, \boldsymbol{\Sigma}_{k}\right)$, where $\boldsymbol{\mu}_{k} \in \mathbb{R}^{D \times 1}$ is the vector of means and $\boldsymbol{\Sigma}_{k} \in \mathbb{R}^{D \times D}$ is the covariance matrix for $k \in\{1, \cdots, K\}$. Let $\mathbf{z} \in \mathbb{R}^{K \times 1}$ be an indicator vector with 1 at its $k^{t h}$ entry (i.e. $\mathbf{z}_{k}=1$ ) and zero otherwise, whenever the variable observed belongs to the $k^{\text {th }}$ Gaussian density. Note that we have $K$ different versions of the $\mathbf{z}$ vector, one for each density. The probability of drawing a variable from the $k^{\text {th }}$ component, or the prior probability of $k$, is denoted as $\pi_{k}:=p\left(\mathbf{z}_{k}=1\right)$. Moreover, the marginal probability for $\mathbf{x}$, obtained marginalizing the joint probability w.r.t. $\mathbf{z}$, is given by

$$
\begin{aligned}
p(\mathbf{x}) & =\sum_{\mathbf{z}} p(\mathbf{z}) p(\mathbf{x} \mid \mathbf{z}) \\
& =\sum_{k=1}^{K} \pi_{k} \mathcal{N}\left(\mathbf{x} \mid \boldsymbol{\mu}_{k}, \boldsymbol{\Sigma}_{k}\right)
\end{aligned}
$$

with prior probabilities satisfying $\sum_{k=1}^{K} \pi_{k}=1$ and $0 \leqslant \pi_{k} \leqslant$ 1. An important quantity is the conditional probability of having observed density $k$ given $\mathbf{x}$, which can be computed using Bayes' theorem as follows

$$
\begin{aligned}
\gamma_{k} & :=p\left(\mathbf{z}_{k}=1 \mid \mathbf{x}\right) \\
& =\frac{\pi_{k} \mathcal{N}\left(\mathbf{x} \mid \boldsymbol{\mu}_{k}, \boldsymbol{\Sigma}_{k}\right)}{\sum_{j=1}^{K} \pi_{j} \mathcal{N}\left(\mathbf{x} \mid \boldsymbol{\mu}_{j}, \boldsymbol{\Sigma}_{k}\right)} .
\end{aligned}
$$

Note that the posterior probability $\gamma_{k}$ computed in (2) gives the information about 'how much' of the $k^{t h}$ density a node is sensing. The value of $\gamma_{k}$ will therefore be helpful to decide the clustering according to which density the observations belong to with highest probability.

The EM algorithm: The EM algorithm is a centralized iterative method that alternates between an expectation (E) step and a maximization (M) step. Consider $N$ independent observation vectors $\mathbf{X}=\left[\mathbf{x}_{1}, \cdots, \mathbf{x}_{N}\right]$ corresponding to each node of the
WSN and define the sets of estimates: $\boldsymbol{\pi}^{t-1}=\left[\pi_{1}^{t-1}, \cdots, \pi_{K}^{t-1}\right]$, $\boldsymbol{\mu}^{t-1}=\left[\boldsymbol{\mu}_{1}^{t-1}, \cdots, \boldsymbol{\mu}_{K}^{t-1}\right], \boldsymbol{\Sigma}^{t-1}=\left[\boldsymbol{\Sigma}_{1}^{t-1}, \cdots, \boldsymbol{\Sigma}_{K}^{t-1}\right]$ at iteration $t-1$. In the E-step of iteration $t$, the algorithm computes the expected log-likelihood of the distribution function using the current estimates, i.e.

$\ln p\left(\mathbf{X} \mid \boldsymbol{\pi}^{t-1}, \boldsymbol{\mu}^{t-1}, \boldsymbol{\Sigma}^{t-1}\right)=\sum_{n=1}^{N} \ln \left\{\sum_{k=1}^{K} \pi_{k}^{t-1} \mathcal{N}\left(\mathbf{x}_{n} \mid \boldsymbol{\mu}_{k}^{t-1}, \boldsymbol{\Sigma}_{k}^{t-1}\right)\right\}$

while in the M-step, new estimates maximizing the function in (3) are derived. For convenience, let's define

$$
N_{k}^{t-1}=\sum_{n=1}^{N} \gamma_{k n}^{t-1}, \quad k=\{1, \cdots, K\}, n=\{1, \cdots, N\}
$$

where $\gamma_{k n}^{t-1}$ is the posterior probability of density $k$ in (2) computed using the estimates from time $t-1$. After convergence of the EM algorithm, the term $N_{k}^{t-1}$ gives the number of nodes observing variables from the $k^{t h}$ density. The values maximizing the log-likelihood function are obtained deriving the expression in (3) w.r.t. $\pi_{k}^{t-1}, \boldsymbol{\mu}_{k}^{t-1}$ and $\boldsymbol{\Sigma}_{k}^{t-1}$ sequentially and equating to zero. Then, the updating values of the M-step are given by

$$
\begin{aligned}
\pi_{k}^{t} & =\frac{N_{k}^{t-1}}{N} \\
\boldsymbol{\mu}_{k}^{t} & =\frac{1}{N_{k}^{t-1}} \sum_{n=1}^{N} \gamma_{k n}^{t-1} \mathbf{x}_{n}
\end{aligned}
$$

and

$$
\boldsymbol{\Sigma}_{k}^{t}=\frac{1}{N_{k}^{t-1}} \sum_{N=1}^{N} \gamma_{k n}^{t-1}\left(\mathbf{x}_{n}-\boldsymbol{\mu}_{k}^{t}\right)\left(\mathbf{x}_{n}-\boldsymbol{\mu}_{k}^{t}\right)^{T} .
$$

Note that the estimate of the mean vector $\boldsymbol{\mu}_{k}^{t}$ in (6) is used in the computation of the sample covariance matrix in (7) at the same iteration.

Assuming that the nodes have some initial estimates $\pi_{k}^{0}, \boldsymbol{\mu}_{k}^{0}$ and $\boldsymbol{\Sigma}_{k}^{0}$ for all $k$, the posterior probabilities for each density can be computed in the E-step using (2). In the M-step, the hidden variables are updated using (5), (6) and (7), continuing with the E-step in (2) until convergence. Observe that the computations of (4), (5), (6) and (7), require global information since the summations are over all the nodes. We are however interested in a distributed implementation of the EM algorithm. In classical consensus ( [7]-[9]) the nodes exchange information locally to reach an agreement regarding an initial value, assuming that they observe the same random variable. In our proposed implementation, the nodes exchange information locally and run the average consensus algorithm to compute the ML estimates of the EM algorithm with properly initialized variables. After convergence of the log-likelihood function, the nodes are able to organize in clusters where the distance measurements are the posterior probabilities of the densities. The advantage is that no knowledge of the network topology is required, as opposed to [3], and there is no need to convey the information to a central node or cluster head, as proposed in [4]. The steps of the algorithm are described in the following section. 


\section{Distributed EM ALGORITHM FOR CLUSTERING}

Consider a WSN composed of $N$ nodes sensing a mixture of $K$ Gaussian densities. The nodes implement the EM algorithm and at iteration $t$, they run the average consensus algorithm to estimate the quantities in (5), (6) and (7). A node $n$ initializes its own multidimensional state $\mathbf{y}_{n}^{t}(l)$ at time $l=0$ of the consensus algorithm and communicates with its nearest neighbors to exchange states, which are updated iteratively according to the difference equation

$$
\mathbf{y}_{n}^{t}(l)=\mathbf{y}_{n}^{t}(l-1)+\epsilon \sum_{j \in \mathcal{N}_{n}(l)}\left(\mathbf{y}_{j}^{t}(l-1)-\mathbf{y}_{n}^{t}(l-1)\right)
$$

where $\mathcal{N}_{n}(l)$ is the instantaneous set of neighbors of node $n$ and $\epsilon$ is chosen to satisfy convergence conditions. In the presence of additive noise, $\epsilon$ can decrease with time to control the noise variance. The convergence of $\mathbf{y}_{n}^{t}(l)$ in (8) is decided using local information only, when its entries are close enough to the entries of $\mathbf{y}_{n}^{t}(l-1)$ such that $\left\|\mathbf{y}_{n}^{t}(l)-\mathbf{y}_{n}^{t}(l-1)\right\|_{\infty} /\left\|\mathbf{y}_{n}^{t}(l)\right\|_{\infty}<\delta$ for a sufficiently small and positive $\delta$. Remark that the index $t$ does not change while running the consensus algorithm. The total number of consensus iterations will depend on the average connectivity $\overline{\mathbf{L}}$, on the connection probability matrix $\mathbf{P}$ and on the value of $\delta$. For instance, a more connected average network will need fewer iterations until convergence than a less connected one for the same value of $\delta$. For a symmetric instantaneous topology, each entry of $\mathbf{y}_{n}^{t}(l)$ in (8) converges asymptotically to the average of the initial values provided that $\epsilon$ is restricted to the interval $\left(0,2 / \lambda_{n}(\overline{\mathbf{L}})\right)[10]$. However, we can just restrict $\epsilon$ to the interval $(0,2 / N]$ when the topology is unknown. The next step consists in defining the set of initial values for each node such that after running consensus they obtain the EM estimates, or a sufficiently good approximation to them.

Since a proper choice of $\epsilon$ guarantees asymptotic convergence to the average of the initial values of (8) as $l \rightarrow \infty$ for a symmetric topology connected on average over time, we need to show that the consensus values approach asymptotically the ML estimates of the EM algorithm. The consensus vector is initialized at node $n$ as follows

$$
\begin{gathered}
\left(\mathbf{y}_{n}^{t}(0)\right)=\left[\gamma_{1 n}^{t-1}, \cdots, \gamma_{K n}^{t-1},\right. \\
\gamma_{1 n}^{t-1} \mathbf{x}_{n}^{T}, \cdots, \gamma_{K n}^{t-1} \mathbf{x}_{n}^{T}, \\
\left(\gamma_{1 n}^{t-1} \operatorname{vec}\left(\mathbf{x}_{n} \mathbf{x}_{n}^{T}\right), \cdots, \gamma_{K n}^{t-1} \operatorname{vec}\left(\mathbf{x}_{n} \mathbf{x}_{n}^{T}\right)\right]^{T}
\end{gathered}
$$

where $\gamma_{k n}^{t-1}$ is computed with (2) for all $k$ using the available estimates $\pi_{k}^{t-1}, \boldsymbol{\mu}_{k}^{t-1}$ and $\boldsymbol{\Sigma}_{k}^{t-1}$. Consider first the distributed estimation of $\pi_{k}^{t}$ for all $k$. The prior probabilities are updated in the $K$ first entries of $\mathbf{y}_{n}^{t}(0)$ given in (9a). After running the consensus, we obtain the updated version of $\pi_{k}^{t}$, since each entry $k$ in (9a) becomes

$$
\frac{1}{N} \sum_{n=1}^{N} \gamma_{k n}^{t-1}=\pi_{k}^{t}
$$

where we have used (5) and substituted for (4). Regarding the estimation of the mean vector $\boldsymbol{\mu}_{k}$ in (6), the next $K \times D$ entries of $\mathbf{y}_{n}^{t}(0)$ are initialized with the entries of the vectors defined in (9b). As $l \rightarrow \infty$ we have

$$
\frac{1}{N} \sum_{n=1}^{N} \gamma_{k n}^{t-1} \mathbf{x}_{n}^{T} .
$$

After reaching consensus, the quantities are divided by the estimate of the prior probability obtained from the corresponding position in the vector $\mathbf{y}_{n}^{t}(l)$, i.e.

$$
\frac{1}{\pi_{k}^{t} N} \sum_{n=1}^{N} \gamma_{k n}^{t-1} \mathbf{x}_{n}^{T}=\frac{1}{N_{k}^{t-1}} \sum_{n=1}^{N} \gamma_{k n}^{t-1} \mathbf{x}_{n}^{T}
$$

which coincides with the estimate of the mean vector in (6). Finally, regarding the estimation of the sample covariance matrix in (7) we initialize the vectors as in (9c). As time evolves, the $K$ quantities in (9c) converge to

$$
\frac{1}{N} \sum_{n=1}^{N} \gamma_{k n}^{t-1} \operatorname{vec}\left(\mathbf{x}_{n} \mathbf{x}_{n}^{T}\right)^{T} .
$$

Rearranging the terms in the summation above back to matrix form, dividing the resulting matrix by $\pi_{k}^{t}$ and subtracting $\boldsymbol{\mu}_{k}\left(\boldsymbol{\mu}_{k}\right)^{T}$ obtained with (9a) and (9b) respectively, we get

$$
\frac{1}{\pi_{k}^{t} N} \sum_{n=1}^{N} \gamma_{k n}^{t-1} \mathbf{x}_{n} \mathbf{x}_{n}^{T}-\boldsymbol{\mu}_{k}^{t}\left(\boldsymbol{\mu}_{k}^{t}\right)^{T}
$$

It is not difficult to check that the expression above is equal to (7).

Summing up, initializing the vector $\mathbf{y}_{n}^{t}(0)$ using (9) and computing (10) and (11) after reaching consensus, we have approximations to the update values of the EM algorithm in (5), (6) and (7) for a given error threshold $\delta$. The choice of $\delta$ will depend on the average connectivity and it controls the trade-off between the final accuracy of the estimation and the power consumption of the WSN, as we will see in the computer simulations in the following section.

\section{Simulations}

We simulate a WSN composed of $N=50$ nodes randomly deployed to sense a mixture of $K=2$ Gaussians, with means $\boldsymbol{\mu}=[2 ; 4]$ and variances $\boldsymbol{\Sigma}=[0.4 ; 0.2]$. The prior probabilities are $\boldsymbol{\pi}=[0.3 ; 0.7]$ and the nodes start with equal initial variables $\boldsymbol{\mu}^{0}=[1 ; 3], \boldsymbol{\Sigma}^{0}=[0.1 ; 0.1]$ and $\boldsymbol{\pi}^{0}=[0.5 ; 0.5]$. Fig. 1 shows the network deployment, where the nodes occupying $70 \%$ of the area, depicted with 'o', are sensing values from density $k=1$, whereas the remaining ones depicted with ' $\square$ ' are sensing density $k=2$. Note that not all nodes sensing the same density are connected to each other. The connection probabilities for the random topology are modeled as r.v.'s uniformly distributed between 0 and 1 and we set $\epsilon=1 / \lambda_{n}(\overline{\mathbf{L}})$. Fig. 2 depicts the log-likelihood function in (3) for the centralized solution versus the distributed implementation with several values of $\delta$ with a fixed topology and $\epsilon=2 /\left(\lambda_{2}(\mathbf{L})+\lambda_{N}(\mathbf{L})\right)$, along with the curve for the packet loss case. For the random packet loss case, the number of consensus iterations is, as expected, higher, and the performance w.r.t the fixed topology is worse due to the fact that a node not receiving any packet 


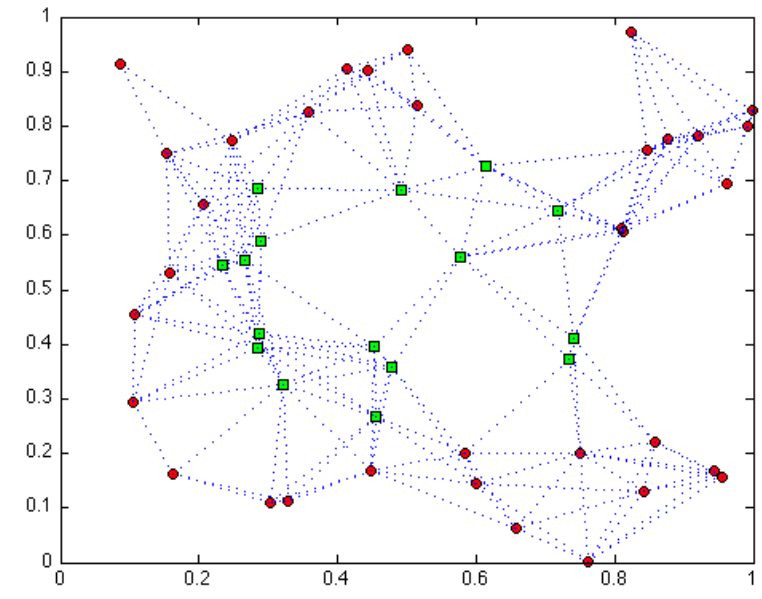

Fig. 1. WSN spatially deployed to sense a mixture of two Gaussians. Different patterns denote different densities being sensed.

from its neighbors will decide for consensus earlier, i.e. with less accurate estimates. Clearly, as $\delta \rightarrow 0$ the consensus values at each EM iteration are closer to the global estimates and the results approximate the centralized solution. However, a small value of $\delta$ means also a high number of consensus iterations, specially in the presence of random link failures. To obtain a trade off between consensus iterations and EM iterations, Fig. 3 depicts the total number of iterations (consensus $\times \mathrm{EM}$ ) as a function of $\delta$, where the nodes take a hard decision for clustering. The results are averaged over 10000 independent realizations, where we have computed the average number of consensus iterations and the average number of EM iterations such that $70 \%$ of the nodes have clustered correctly for a given $\delta$. We observe that the minimum is attained when $\delta \approx 0.5$ approximately. In other words, a high accuracy of the consensus values is not needed for clustering in this deployment. Allowing a higher error for the estimates, the nodes are able to cluster correctly while reducing the overall energy consumption of the WSN. For higher values of $\delta$, we have observed that the percentage of correct clustering does not increase with the number of EM iterations.

\section{CONCLUSiOnS}

We have effectively implemented the EM algorithm in a distributed fashion without the necessity to convey the information to a central unit or a cluster head, using the simplicity of the consensus algorithm and initializing the variables in a proper manner. The algorithm shows robustness w.r.t. packet loss and random communication failures.

\section{REFERENCES}

[1] C. M. Bishop, Pattern recognition and machine learning, M. Jordan, J. Kleinberg, and B. Schlkopf, Eds. Springer, 2006.

[2] D. J. MacKay, Information Theory, Inference, and Learning Algorithms. Cambridge University Press, 2003.

[3] R. D. Nowak, "Distributed EM algorithms for density estimation and clustering in sensor networks," IEEE Transactions on Signal Processing, vol. 51, no. 8, pp. 2245-2253, 2003.

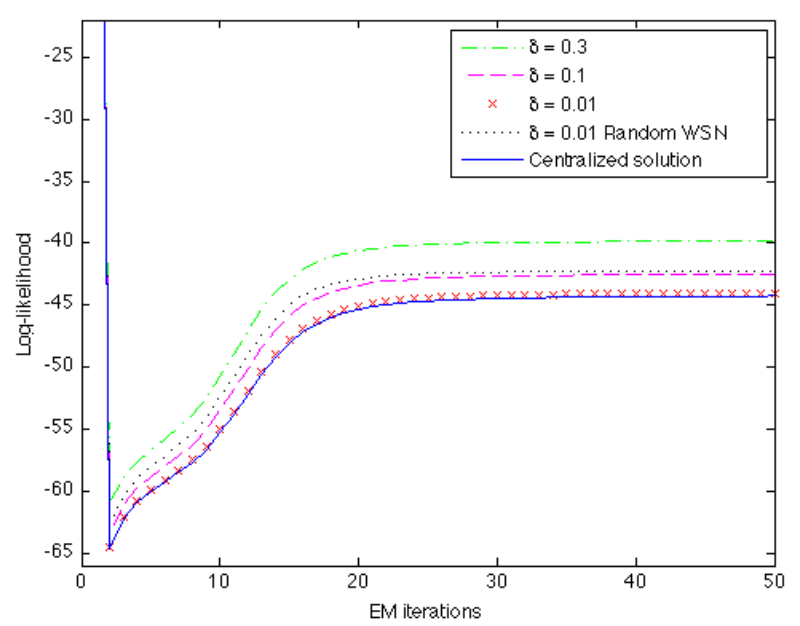

Fig. 2. Log-likelihood function for different values of $\delta$.

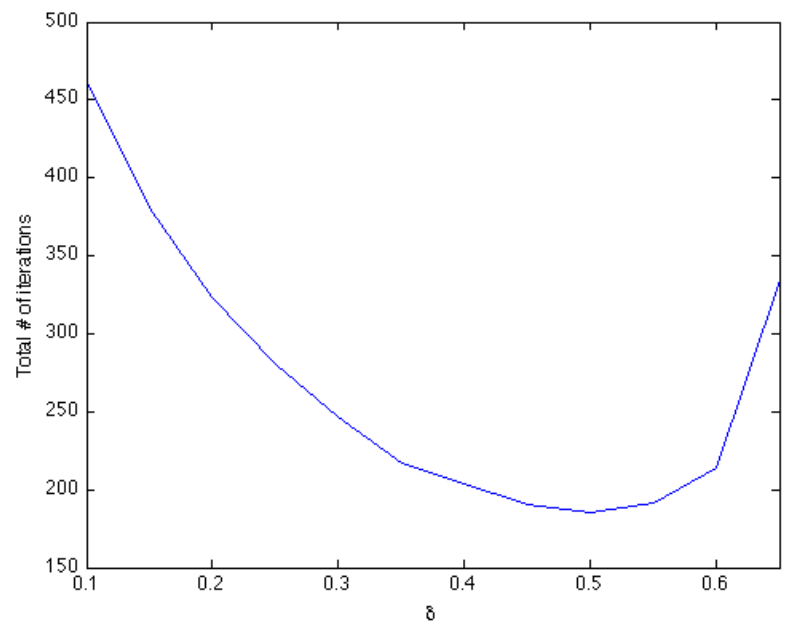

Fig. 3. Iterations needed for $70 \%$ correct clustering as a function of $\delta$.

[4] P. A. Forero, A. Cano, and G. B. Giannakis, "Consensus-based distributed expectation-maximization algorithm for density estimation and classification using wireless sensor networks," in Proc. IEEE International Conference on Acoustics, Speech and Signal Processing ICASSP 2008, 2008, pp. 1989-1992.

[5] C. Godsil and G. Royle, Algebraic graph theory. Graduate Texts in Mathematics. Berlin, Germany: Springer-Verlag, 2001, vol. 207.

[6] R. Horn and C. Johnson, Matrix analysis. Cambridge University Press, 2006.

[7] R. Olfati-Saber and R. Murray, "Consensus problems in networks of agents with switching topology and time-delays," IEEE Transactions on Automatic Control, vol. 49, no. 9, pp. 1520-1533, Sept. 2004.

[8] D. Scherber and H. Papadopoulos, "Distributed computation of averages over ad hoc networks," IEEE Journal on Selected Areas in Communications, vol. 23, no. 4, pp. 776-787, April 2005.

[9] S. Barbarossa and G. Scutari, "Decentralized maximum-likelihood estimation for sensor networks composed of nonlinearly coupled dynamical systems," IEEE Transactions on Signal Processing, vol. 55, no. 7, pp. 3456-3470, 2007.

[10] S. Kar and J. Moura, "Distributed average consensus in sensor networks with random link failures," in IEEE International Conference on Acoustics, Speech and Signal Processing ICASSP, vol. 2, 15-20 April 2007, pp. II-1013-II-1016. 